\title{
Boosting Solar Steam Generation by Using AIE Photothermal Molecule-Doped 3D Nanofibrous Aerogel with Self-Pumping Water Function
}

\author{
Haoxuan $\mathrm{Li}^{\mathrm{a}, \mathrm{b}, \mathrm{c}}$, Haifei Wen ${ }^{\mathrm{a}}$, Jie Li ${ }^{\mathrm{a}, \mathrm{b}}$, Jiachang Huang ${ }^{\mathrm{a}, \mathrm{b}, \mathrm{c}}$, Dong Wang**a ${ }^{\text {a }}$, Ben Zhong Tang*c \\ a Centre for AIE Research, College of Material Science and Engineering, Shenzhen University, \\ Shenzhen 518061, P. R. China \\ E-mail: wangd@szu.edu.cn \\ $b$ Key Laboratory of Optoelectronic Devices and Systems of Ministry of Education and \\ Guangdong Province, College of Optoelectronic Engineering, Shenzhen University, Shenzhen \\ 518061, P. R. China \\ c Department of Chemistry, The Hong Kong University of Science and Technology, Clear Water \\ Bay, Kowloon, Hong Kong, China \\ E-mail:tangbenz@ust.hk
}




\begin{abstract}
Utilizing solar energy to generate clean water by interface solar steam generation is considered to be a promising strategy to address the challenge of water shortage globally. However, high evaporation rate and long-term sustainability have rarely been achieved simultaneously, due to salt accumulation, discontinuous water supply and insufficient photothermal conversion. Herein, we demonstrate that a three-dimensional nanofibrous aerogel (3D NA) with Janus layers enables floating on the surface water by hydrophobic layer and continues pumping water by hydrophilic layer and interconnected porous structure. More notably, an aggregation-induced emission (AIE) photothermal molecule is doped into nanofibers for the first time, which was endowed with superior capacity of transferring solar energy into heat. Combining these unique benefits, the presented 3D NA exhibits extremely high evaporation rate $\left(1.99 \mathrm{~kg} \mathrm{~m}^{-2} \mathrm{~h}^{-1}\right)$ and solar-to-vapor conversion efficiency ( $89 \%)$ under irradiation of 1 sun. Besides, there is no significant change in evaporation performance after 21 cycles in the case of seawater treatment, suggesting that the designed 3D NA possess sustainable stability and self-cleaning function to restrain salt deposition. With highly efficient evaporation rate and long-term sustainable solar steam generation, such 3D NA can offer new strategy for desalination and sewage treatment.
\end{abstract}

Key works: Solar desalination; Aggregation-induced emission; 3D nanofibrous aerogel; Electrospinning 


\section{Introduction}

With the rapid developments of urbanization and industrialization, the fast-growing freshwater demand and the limited available water resource have become serious global challenges. ${ }^{1,2}$ While, seawater, making up total $97.5 \%$ of water resources in earth, is a kind of seemingly inexhaustible resource for water, hence seawater desalination has been acknowledged as the most applicable route for supplying a large amount of freshwater to meet the requirement of life and modern industry. ${ }^{3-5}$ Nowadays, various technologies of seawater desalination, such as reverse osmosis (RO) and ultrafiltration, have been developed to obtain clean water from sea. ${ }^{6,7}$ However, both the requirement of a high pressure to drive the filtering and short service life of RO/ultrafiltration membrane made these technologies have to face the challenge of costly and cumbersome operations. ${ }^{8,9}$ Besides, the majority of state-of-the-art water treatment factories are energy- and capital-intensive, the limitation of their application to alleviate the world's water shortage problem is still not addressed. ${ }^{10}$ In the circumstances, interfacial solar vapor generation, which can efficiently convert solar energy to heat and inspire seawater vaporization, has been considered as an economic and sustainable strategy to solve growing freshwater scarcity. ${ }^{11-13}$

Solar driven vapor opens up new perspectives for seawater desalination due to the abundance of solar energy as well as almost zero carbon emission when treating seawater, even wastewater. $^{14,15}$ Solar steam efficiency and solar steam sustainability are two key criterions to evaluate solar desalination systems. High solar steam efficiency could be realized by the utilization of advanced materials contains good photothermal conversion performance and floating smart structure with well-designed water transportation and heat flow. ${ }^{16}$ Recently, various photothermal materials, including carbon-based materials, ${ }^{17-19}$ plasmonic-based absorbers ${ }^{20,21}$ as well as organic absorbers, ${ }^{22,23}$ have been investigated to absorb solar energy and transfer it to thermal energy for heating water. However, in most if not all of the current progress, high solar steam efficiency (>85\%) has been rarely achieved under 1 sun irradiation, which severely impede the scalable application. ${ }^{24,25}$ Aiming to boost solar steam generation, utilization of well-performed photothermal conversion agent is one of highly desirable strategies. In this 
related context, as a newly emerged photothermal agent, well-tailored aggregation-induced emission (AIE)-active molecule could be a promising candidate. ${ }^{26-29}$ AIE molecules are often propeller-like in shape and inherently possess, therefore, efficient molecular mobility ${ }^{30}$, which facilitates non-radiative decay of excitons to significantly convert light energy into heat. ${ }^{31,32}$ Moreover, compared with traditional photothermal materials, such as carbon or plasmonic agent, an unparalleled advantage is that the AIE molecules generally have good solubility in common organic solvents, endowing them with excellent workability for smart materials. To the best of our knowledge, there have been no previous reports on utilizing photothermal AIE molecules in solar driven vapor for seawater desalination. Additionally, it has been demonstrated that the floating smart structure, such as carbon foams ${ }^{33,34}$, AAO substrates ${ }^{35}$, natural wood ${ }^{36-38}$ or plant $^{39}$ and hydrogel ${ }^{40,41}$, are capable of pumping water to heating region by capillary wicking effect, resulting in good solar steam efficiency. However, high efficiency and long-term sustainability of solar steam are hard to achieve simultaneously. As water evaporation rate is more quickly than water supply, the salt will be left behind. While, salt accumulation will shelter from sunlight and block interior porous for water supply and vapor release, which significantly degrades the sustainability of solar evaporation. ${ }^{42,43}$

Herein, we propose a facile protocol to fabricate a three-dimensional nanofibrous aerogel (3D NA) doping with AIE photothermal molecules for solar steam generation. The nanofibrous aerogel has porous hydrophilic/hydrophobic Janus layers, which enable it floating on the surface water by hydrophobic layer, and continuously self-pumping water by hydrophilic layer and the interconnected porous structure. By utilization of this aerogel, efficient and sustainable solar desalination was successfully realized under normal 1 sun irradiation without extra supporting systems, making it greatly promising to improve the scalability and feasibility of solar desalination technology.

\section{Results and Discussion}

Fabrication of 3D NA with Janus layer. Figure. 1 shows the concept of 3D NA with Janus layers for effective solar steam generation. Poly(vinylidene fluoride -co-hexafluoropropylene) 
(PVDF-HFP) nanofibers were selected as the major part to construct the 3D NA, and an AIE-active molecule MTTT-BT was blended with PVDF-HFP nanofibers as photothermal agent. Low melt point polycaprolactone (PCL) nanofibers were introduced into 3D NA as thermal welding medium to enhance their structural stability. The as-prepared electrospun MTTT-BT-contained PVDF-HFP and PCL nanofibrous mats were blended in a water/tert-butanol $(4: 1, \mathrm{v} / \mathrm{v})$ mixture solution and then to form well-dispersed short nanofibers dispersions and freeze dried in to a 3D NA. A single layer hydrophilic treatment of the 3D NA was conducted by plasma exposure and gelatin electrospraying successively. The plasma exposure time and electrospraying time was set to $3 \mathrm{~min}$ and $30 \mathrm{~min}$, respectively. During electrospraying on the exposure layer, gelatin nanoparticles immediately spread and permeate into the hydrophilic nanofibers in 3D NA. With increasing the depth, the 3D NA exhibit gradient weakened hydrophilic of PVDF-HFP, which can prevent the gelatin nanoparticles spreading deeper. After cross-linking via expose to glutaraldehyde vapor, the temporary hydrophilicity of the 3D NA achieved by plasma treatment turns permanent. The thickness of each layer can be regulated by modulating plasma or electrospray time. ${ }^{44}$ After the plasma and electrospraying treatments, the 3D NA with Janus layer of hydrophobic and hydrophilic layer was achieved and utilized for solar steam generation.

As depicted in Fig. 2A, AIE photothermal molecule MTTT-BT was facilely synthesized by two-step reactions. In the primary step, Suzuki-Miyaura coupling reaction smoothly proceeded by employing (4-(bis(4-methoxyphenyl)methyl)phenyl)boronic acid and 5'-bromo-[2,2'-bithiophene]-5-carbaldehyde as starting materials, producing intermediate product 1 with the yield of $73 \%$. Condensation reaction of 3-ethyl-2-methylbenzothiazolium iodide with 1 was then conducted to obtain the desired compound MTTT-BT with the yield of $83 \%$. The UV-Vis spectrum of MTTT-BT in the DMSO shows that the absorption wavelength is mainly located at 300-750 nm with a molar absorption coefficient of $8.37 \times 10^{4} \mathrm{~L} \mathrm{~mol}^{-1} \mathrm{~cm}^{-1}$ at the absorption maximum of $560 \mathrm{~nm}$ (Fig. 2B). The strong and broad absorption is beneficial to absorb light energy of sun light. Subsequently, the AIE features of MTTT-BT were further 
evaluated by mixed solvent system of DMSO and toluene with different toluene fractions. With raising the toluene fraction, the fluorescence emission intensity of MTTT-BT gradually enhanced due to the formation of nanoaggregates. As the toluene fraction increased to $90 \%, 95 \%$ and $99 \%$, the emission increased significantly, which were about 7, 14.7 and 25.3 times higher than those in pure DMSO solution, revealing typical AIE features (Fig. 2 C and D). Such phenomenon could be attributed to the restriction of rotation motions upon the formation of aggregates. Moreover, the AIE photothermal agent possesses a rotor-like twisted structure with loose packing, where molecular motion is still active, would favorable to generate heat in nanofibers (aggregates condition). Furthermore, the photothermal conversion capability of MTTT-BT was assessed. Upon solar irradiation of 1 sun, the temperature of MTTT-BT powder increases with time and reach a maximum of $43.1^{\circ} \mathrm{C}$ within $150 \mathrm{~s}$, suggesting its good capacity of transferring solar energy to heat (Supplementary Fig. 1). Moreover, comparing with previously reported photothermal materials, such as carbon nanotubes, MTTT-BT exhibit much better solubility in organic solvent, which is favor to dope MTTT-BT into electrospun nanofibers. The digital photograph and scanning electron microscope (SEM) images taken from PVDF-HFP/MTTT-BT nanofibrous mat with different contents of MTTT-BT were shown in Supplementary Fig. 2. PVDF-HFFP/MTTT-BT nanofibers with $1 \%, 5 \%$ and $10 \%$ of MTTT-BT were successfully fabricated and displayed smooth surface and continuous structure. No significant change in morphology of the nanofibers was observed, even the content of MTTT-BT was increased to $10 \%$, owing to the excellent fusion of MTTT-BT molecules with PVDF-HFP. Upon solar irradiation of 1 sun, the temperature of MTTT-BT/PVDF-HFP nanofibrous mats with 1\%, 5\% and $10 \%$ of MTTT-BT reach the maxima at $42.6,46.1$ and $46.4{ }^{\circ} \mathrm{C}$, respectively. Hence, MTTT-BT/PVDF-HFP nanofibrous mats with 5\% of MTTT-BT were chosen to fabricate nanofibers-based 3D NA.

Recent progress on fabricating 3D NA provides a new strategy to design absorber or matrix for solar steam generation. ${ }^{45}$ Nevertheless, achieving directional fluid transport in 3D NA remains a challenge, because water cannot be pumped up to the surface of the 3D NA only by 
capillarity effect of porous structure. In this study, the combination of plasma treatment and electrospraying methods enables the 3D NA to possess permanent Janus surface, which is capable of boosting directional fluid transport of water from hydrophobic layer to hydrophilic layer. Significantly, the hydrophobic bottom layer can support 3D NA in floating on the surface of water, making 3D NA highly efficient for capturing solar energy (Fig. 3A and 3B). While, the hydrophilic upper layer is beneficial to pump water for evaporation resulting from the superior wicking and capillarity effect. As illustrated in Fig. 3B, water can be efficiently pumped up though interconnected porous of 3D NA, and then spread the entire surface. Therefore, the 3D NA can be supplied with continuous water flow without direct contact with bulk water, resulting excellent localized heating at the air-liquid interface. The hierarchically porous structure of the presented 3D NA with highly interconnected micropores and nanopores provide micro channels for water transportation and vapor release (Fig. $3 \mathrm{C}$ and D). In addition, such porous structure with high porosity displays low thermal conductivity, suggesting their promising thermal insulation for solar steam performance. The compress stress strain curves revealed that the 3D NA can undergo a compressive stress as high as $1.12 \mathrm{kPa}$, and $33.8 \%$ of compressive strain, endowed robust and good stability for recovering after large deformations (Fig. 3E). Moreover, the 3D NA can be easily scaled up via using a large molding mould and provided various shapes, including heart and circles shapes even alphabet of AIE (Fig. 3F and Supplementary Fig. 3), which has photothermal imaging capacity under the irradiation of sunlight owing to its good photothermal conversion performance (Fig. 3G). Interestingly, such 3D NA has an ultralow density of about $10 \mathrm{mg} \mathrm{cm}^{-3}$ and can stand on the tip of a rabbit hair (Fig. 3H). Comparing with these previously reported solar absorbing materials for solar steam generation, the 3D NA holds the lowest density, benefiting from its nanofibrous based 3D structure with macro and nanopores. Such ultralight 3D NA minimizes the amount of AIE agent and avoids volumetric heating.

Photothermal effect. The UV-vis-NIR absorption spectrum was shown in Fig. 4A, the 3D NA exhibits a strong and broad light absorption across the solar spectrum with 200-2500 nm of wavelength. While, the PVDF-HFP nanofibrous mats containing 5\% and $10 \%$ of MTTT-BT 
showed efficient absorption at $300-750 \mathrm{~nm}$, which is characteristic of MTTT-BT, suggesting that the highly interconnected porous structure of the 3D NA is favorable to increase the optical path and decrease the reflection. Prior to the test of solar steam generation, the photothermal performance of the 3D NA in dry state was investigated under constant solar irradiation at a power density of $1 \mathrm{~kW} / \mathrm{m}^{2}$ (1 sun) (Fig. 4B). It was observed that the surface temperature of the 3D NA significantly reached to $87^{\circ} \mathrm{C}$ upon 100 s solar irradiation, which is much higher than that of the pristine PVDF-HFP nanofibrous mat with 5\% of MTTT-BT under the same irradiation conditions, implying that the 3D NA possess excellent solar absorbance and heat transfer capacities. The inferior photothermal conversion output of PVDF-HFP/MTTT-BT nanofibrous could result from the dense structure of electrospun nanofibrous mat. The extraordinary capability of the 3D NA for photothermal conversion was further confirmed under different irradiation powers, as shown in Fig. 4C and Supplementary Fig.4, the surface temperature of the 3D NA was determined to be 106.5 and $119.8{ }^{\circ} \mathrm{C}$ under irradiation of 2 and 3 sun, respectively. Figure. 4D shows the temperature at different positions of 3D NA under 1 sun irradiation, to be noted here, the 3D NA was not wrapped by thermal insulation materials such as foam. With the design of the highly porous 3D structure, the solar energy was highly localized on the surface of the 3D NA, where the temperature increased to $87^{\circ} \mathrm{C}$ within $100 \mathrm{~s}$. While there was only $3{ }^{\circ} \mathrm{C}$ increase in temperature on bottom of the 3D NA comparing with ambient temperature $\left(32{ }^{\circ} \mathrm{C}\right)$. As a result of this, the 3D NA is able to significantly suppress heat loss from evaporate area to bulk water, enhancing the efficiency of solar steam generation.

Solar steam generation performance. In order to evaluate the practical application of 3D NA for solar steam generation, simulated seawater with $3.5 \mathrm{wt} \%$ of $\mathrm{NaCl}$ was utilized for test. As the 3D NA floating on simulated seawater, then seawater was pumped and spread to entire evaporation area. Once the irradiation is turned on, the hydrophilic layer absorbs solar and transfer it to heat, further heating the pumped seawater and generating vapor. As shown in Fig. $5 \mathrm{~A}$, the temperatures on the surface of $3 \mathrm{D} \mathrm{NA}$ were up to $41.2,50.7$ and $59.8{ }^{\circ} \mathrm{C}$ under irradiations of 1, 2 and 3 sun for $100 \mathrm{~s}$, respectively. The lower temperatures than that in dry 
condition can be attributed to the tremendous thermal energy consumption for heating seawater. Within prolonged irradiation, the temperature trend to be constant, indicating an energy balance of heating water with steam generation. As shown in Supplementary Fig. 5, at 3 sun, even 2 sun, steam is generated. It was clearly found that the evaporation rates of 3D NA reach constant values after 30 min irradiation, which are 1.99, 3.24 and $4.31 \mathrm{~kg} \mathrm{~m}^{-2} \mathrm{~h}^{-1}$ under different solar of 1 , 2 and $3 \mathrm{~kW} \mathrm{~m}^{-2}$, respectively (Fig.5 B and C). The conversion efficiency of solar to steam generation is defined as the following formula ${ }^{34,46}$ :

$$
\eta=m h_{L V} / C_{o p t} P_{0}
$$

where $m$ is the mass flux, $h_{L V}$ is the latent enthalpy of liquid to vapor phase change, $C_{o p t}$ is the optical concentration and $P_{0}$ is constant solar of 1 sun. Figure. 5C shows the evaporation rate under steady state and calculated solar conversion efficiencies of 3D NA. The conversion efficiency of 3D NA gradually increased from $89.0 \%$ to $91.3 \%$ and $94.7 \%$, with increasing irradiation intensity from 1 to 2 and 3 sun. Notably, in other state-of-the-art studies, such a high efficiency (greater than $85.0 \%$ ) is difficult to achieve under 1 sun. Sustainability is another key criterion to assess solar desalination systems. Figure. 5D shows the evaporation rate change of the 3D NA under fixed solar irradiation ( 1 and 3 sun), and the time was set to $8 \mathrm{~h}$, which is close to irradiation period of sunlight in one day. It was found that the evaporation rate of the 3D NA under 1 sun of irradiation retains invariant, which is favor to sustainable and stable manipulation at daytime. While, the evaporation rate under 3 sun of irradiation slightly decreased after $7 \mathrm{~h}$, due to the deposition of salt on the surface. Nonetheless, the salts deposited on the surface of 3D NA could dissolve completely in $30 \mathrm{~min}$ after turning off the irradiation, because the fresh pumped seawater can dissolve the accumulated salts and return it to the surrounding seawater after turning off the irradiation (Supplementary Fig. 6). To further explore the self-cleaning capacity of 3D NA after desalination, SEM images of 3D NA at $7 \mathrm{~h}$ under irradiation of 3 sun and $0.5 \mathrm{~h}$ after turning off the irradiation. A thick layer of $\mathrm{NaCl}$ crystal was observed on the surface of the 3D NA after $7 \mathrm{~h}$ of irradiation (Supplementary Fig. 7A), led to a decline in the evaporate rate. However, after turning off irradiation, no salt could be observed on the surface of the nanofibers 
in $0.5 \mathrm{~h}$ (Supplementary Fig. 7B), suggesting excellent self-cleaning property. Interestingly, there was no salts deposition on the surface of 3D NA under 1 sun of irradiation, the reason can be explained that the rate of salts deposition is slower than pumped seawater rate. No accumulation of salt on the surface was observed on the surface, even the concentration of $\mathrm{NaCl}$ was increased to $20 \mathrm{wt} \%$ (near to saturated solution) (Supplementary Fig. 8), strongly indicating the great potential of this solar desalination system for the practical application on seawater. The efficient self-cleaning function of salt deposition can be attributed to the unique structural features of the 3D NA with Janus layer, including (1) the interconnected porous (micropore and nanopore) throughout hydrophilic layer and hydrophobic layer, as well as (2) the continuously pumped seawater by wicking and capillarity effect provides seawater flowing that ensures efficient mass exchange for rapid dissolution of the deposited salt. ${ }^{47}$ In this way, the presented 3D NA can even work under high intensity of irradiation on daytime, and self-clean on night, so the salt blockage issue during steam generation can be perfectly solved. Furthermore, cycling performance was investigated for charactering the sustainable capacity of the 3D NA. As illustrated in Fig. 5E, after 21 cycles under alternate 1 and 3 sun of irradiation, the evaporation rate stays the same as the first cycle under 1 sun, indicating excellent stability and sustainability. Remarkably, the comparison with the state-of-the-art reported solar desalination systems solidly suggests that the presented 3D NA achieves both outstanding solar steam efficiency and excellent solar steam sustainability (Fig. 5F).

As a proof-of-concept, we designed a solar steam generation prototype made of PMMA organic glass with $92 \%$ of transmittance, and water chute was attached on the inner of the glass for collecting the condensational steam. In experiment, 3D NA with a diameter of $2 \mathrm{~cm}$ was floating on a beaker of simulated seawater contains $3.5 \mathrm{wt} \%$ of $\mathrm{NaCl}$, which was placed into the bottom of the prototype (Fig. 6A). The outdoor experiment on the roof was carried out from 05:00 to 19:00 under natural sunlight with a solar flux of maximum value at $0.65 \mathrm{kw} \mathrm{m}^{-2}$. As shown in Fig. 6B, the evaporation rate of the 3D NA at 06:00 was only $0.17 \mathrm{~kg} \mathrm{~m}^{-2} \mathrm{~h}^{-1}$, which agrees well with the dark-condition data. With time elapsing, the evaporation rate increased from 
$0.74 \mathrm{~kg} \mathrm{~m}^{-2} \mathrm{~h}^{-1}$ at 08:00 am to $1.90 \mathrm{~kg} \mathrm{~m}^{-2} \mathrm{~h}^{-1}$ at 14:00, and then decreased to $1.56 \mathrm{~kg} \mathrm{~m}^{-2} \mathrm{~h}^{-1}$ at 18:00. The average seawater evaporation rate was calculated to $1.52 \mathrm{~kg} \mathrm{~m}^{-2} \mathrm{~h}^{-1}$, indicating an enhanced evaporation, compared with that in simulated test conditions, mainly because the high ambient temperature (reached to $35^{\circ} \mathrm{C}$ ) could preheat the bulk seawater. As a result of this, the 3D NA process a capability to achieve about $15.2 \mathrm{~kg} \mathrm{~m}^{-2} \mathrm{~d}^{-1}$ of clean water with long term of work time under natural sunlight in theory, which is considerable yield of clean water generated by natural sunlight without solar concentrator (Fig. 6C). With the unique multiple functions in the aspect of Janus layer, interconnected porous as well as highly photothermal conversion capacity, we envision that the developed 3D NA will open up a new way for membrane-less and low energy consume for seawater desalination.

\section{Conclusions}

To sum up, we demonstrate for the first time the design and preparation of a Janus 3D nanofibrous aerogel with hierarchical structure built up by PVDF-HFP nanofibers and AIE photothermal molecule, and its application for solar desalinization. The presented aerogel possesses various impressive characteristics, such as high solar-to-heat conversion, excellent wicking and capillarity effect, well-designed localized heating on its surface and interconnected porous networks. All these features endow the 3D NA with as high as $1.99 \mathrm{~kg} \mathrm{~m}^{-2} \mathrm{~h}^{-1}$ of evaporation rate, up to $89 \%$ solar-to-vapor conversion efficiency under irradiation of 1 sun, as well as a supremely high yield $\left(15.2 \mathrm{~kg} \mathrm{~m}^{-2} \mathrm{~d}^{-1}\right)$ of clean water under natural sunlight. The excellent performance in solar vapor generation and other distinct advantages including sustainable stability and self-cleaning function, make the 3D NA with highly tailored properties superior to the previously reported materials. This work will offer a blueprint for the next generation of solar desalination systems with high efficiency and sustainability, and stimulate the development of sustainable and green water treatment industry.

\section{Methods}

Chemicals and materials. Poly(vinylidene fluoride -co-hexafluoropropylene) (PVDF-HFP) ( $\mathrm{Mw}=40$ 000), polycaprolactone (PCL, average $\mathrm{Mn}=80000$ ), tetrahydrofuran (THF), $N$, 
$N$-dimethylformamide (DMF), and were all obtained from Sigma-Aldrich (St. Louis, MO, USA). Preparation of MTTT-BT/PVDF-HFP and PCL nanofibrous mat. For electrospun MTTT-BT/PVDF-HFP nanofibers, the solution for electrospinning was prepared by dissolving $1.8 \mathrm{~g}$ of PVDF-HFP in $10 \mathrm{~mL}$ mixture of THF and DMF (v/v = 3:7) at a final concentration of 18 wt.\%, and then $90 \mathrm{mg}$ of MTTT-BT powders were added into the as-prepared solution. For PCL nanofibers, the solution for electrospinning was prepared by dissolving $1.2 \mathrm{~g}$ of PCL in $10 \mathrm{~mL}$ mixture of $\mathrm{THF}$ and $\mathrm{DMF}(\mathrm{v} / \mathrm{v}=3: 7)$ at a final concentration of 12 wt.\%. Both electrospun PVDF-HFP and PCL nanofibers were performed using same setup and parameter. The solution was loaded into a 5-mL plastic syringe with a 21-gauge needle attached and dispensed using a KD Scientific syringe pump. The injection rate was set at $0.8 \mathrm{~mL} / \mathrm{h}$. A voltage of $15 \mathrm{kV}$ generated by Gamma High Voltage was applied to the needle, while the distance between the tip of the needle to the collector was set to $15 \mathrm{~cm}$.

Fabrication of 3D NA with Janus layer. In typical method for the fabrication of 3D NA were prepared as described previously. ${ }^{[45]}$ In case of 3D NA with a density of $10 \mathrm{mg} \mathrm{cm}^{-3}, 0.75 \mathrm{~g}$ of MTTT-BT/ PVDF-HFP nanofibers and $0.25 \mathrm{~g}$ of PCL nanofibrous mat were cut into strips, and then placed into high speed blender (MJPBJ01YM) (MI, China) contains $100 \mathrm{~mL}$ of water/tert-butanol mixture $(4: 1, w / w)$, achieving uniform short nanofibers dispersions. Next, the dispersions were poured into the mould and frozen in liquid nitrogen for $30 \mathrm{~min}$, and then freeze dried in Gold SIM PF-5 freeze dryer for $48 \mathrm{~h}$ to get the 3D NA. In order to enhance the stability of the $3 \mathrm{D} \mathrm{NA}$, they were putted into oven heated to $100{ }^{\circ} \mathrm{C}$ for $30 \mathrm{~min}$ to construct the connection between nanofibers. Finally, to obtain the 3D NA with Janus layer, a side layer of the 3D NA was exposed by oxygen plasma and then electrosprayed by gelatin solution. The electrospraying solution was prepared dissolving $0.5 \mathrm{~g}$ of gelatin (fish skin, Sigma) in $10 \mathrm{~mL}$ of ethanol at a concentration of $50 \mathrm{mg} / \mathrm{mL}$. The electrospraying was performed using electrospinning setup, and the voltage and the pumping rate were set to $20 \mathrm{kV}$ and $1 \mathrm{~mL} / \mathrm{h}$. The plasma exposure time and electrospraying time was set to $3 \mathrm{~min}$ and $30 \mathrm{~min}$, respectively. During electrospraying on the exposed layer of the 3D NA, gelatin nanoparticles immediately spreads 
and permeates into the hydrophilic nanofibers in 3D NA. With the increase deep of 3D NA, the nanofibers exhibit gradient weak hydrophilic and even hydrophobic nature of PVDF-HFP in final, which can prevent the gelatin nanoparticles spreading deeper. After cross-linking via expose to glutaraldehyde vapor for $4 \mathrm{~h}$, the temporary hydrophilic of the 3D NA achieved by plasma treatment turns permanent.

Characterization. The SEM images of samples were observed using a SU-70 field emission SEM (Hitachi, Japan). Water contact angle were measured by a Data-Physics OCA 15EC at room temperature. Compressive mechanical properties were measured using an WDW-20 electromechanical universal testing machine (Shanghai Hualong Test Instruments Co., China). The prepared samples $(\mathrm{n}=3)$ were cut into testing strips of $5 \mathrm{~cm} \times 5 \mathrm{~cm}$ and compression speed was $0.5 \mathrm{~mm} / \mathrm{min}$. The IR thermal images and digital photographs were taken by E6 IR-camera (FLIR, USA) and mate-20 pro mobile phone (HUAWEI, China), respectively. Absorbance spectra and AIE curves were recorded using PerkinElmer Lambda 950 (PERKINELMER, USA) and FS 5 fluorescence spectrometer (Edinburgh instruments, UK).

Solar steam generation measurements. The experiment of solar steam generation was performed by a Solar-500L solar simulator system (NBET, Beijing), contains a solar simulator and an AM 1.5G light filter. The solar density is calculated by a VLP-2000 light power meter (LASER, Changchun). A 3D NA was floated on the water in a glass beaker that was placed in the irradiation area of the simulated solar. As the water spread over the surface of the 3D NA, the irradiation was turned on and the mass change was measured via a PR224ZH high accuracy balance (OHAUS, New Jersey).

\section{Reference}

[1] Vorosmarty, C. J., Green, P., Salisbury, J. \& Lammers, R. B. Global water resources: vulnerability from climate change and population growth. Science $\mathbf{2 8 9}$, 284-288 (2000).

[2] Rodell, M. et al. Emerging trends in global freshwater availability. Nature 557, 651-659 
(2018)

[3] Ma, Q. et al. MOF-based hierarchical structures for solar-thermal clean water production. $A d v$. Mater. 31, 1808249 (2019).

[4] Tan, Z. et al. Polyamide membranes with nanoscale Turing structures for water purification. Science 360, 518- 523 (2018).

[5] Shannon, M. et al. Science and technology for water purification in the coming decades. Nature 452, 301-310 (2008).

[6] Werber, J, R., Osuji, C, O. \& Elimelech, M. Materials for next-generation desalination and water purification membranes. Nat. Rev. Mater. 1, 1-15 (2016)

[7] Elimelech, M. \& Phillip, W, A. The future of seawater desalination: energy, technology, and the environment. Science 333, $712-717$ (2011).

[8] Gude, V, G. Desalination and sustainability- an appraisal and current perspective. Water research 89, 87-106 (2016).

[9] Jeon, S. et al. Desalination via a new membrane capacitive deionization process utilizing flow-electrodes. Energy Environ. Sci. 6, 1471-1475 (2012)

[10] Cheng X, Q. et al. Towards sustainable ultrafast molecular-separation membranes: from conventional polymers to emerging materials. Prog. Mater. Sci. 92, 258-283 (2018)

[11] Tao, P. et al. Solar-driven interfacial evaporation. Nat. Energy 3, 1031-1041 (2018).

[12] Gao, M. M. et al. Solar absorber material and system designs for photothermal water vaporization towards clean water and energy production. Energy Environ. Sci. 12, 841-864 (2019).

[13] Zhou, L. et al. The revival of thermal utilization from the sun: interfacial solar vapor generation. Natl. Sci. Rev. 6, 562-578 (2019)

[14] $\mathrm{Xu}, \mathrm{N}$. et al. A water lily-inspired hierarchical design for stable and efficient solar evaporation of high-salinity brine. Sci. Adv. 5, eaaw7013 (2019).

[15] Li, X. et al. Graphene oxide-based efficient and scalable solar desalination under one sun with a confined 2D water path. Proc. Natl. Acad. Sci. USA 113, 13953-13958 (2016). 
[16] Zhu, L. et al. Recent progress in solar-driven interfacial water evaporation: advanced designs and applications. Nano energy 57, 507-518 (2019).

[17] Li, Y. et al. 3D-printed, All in one evaporator for high efficiency solar steam generation under 1 sun illumination. Adv. Mater. 29, 1700981 (2018)

[18] $\mathrm{Hu}, \mathrm{X}$. et al. Tailoring graphene oxide-based aerogels for efficient solar steam generation under one sun. Adv. Mater. 29, 1604031 (2017).

[19] Li, X. et al. Enhancement of interfacial solar vapor generation by environmental energy. Joule 2, 1331-1338 (2018).

[20] Gao, M, Connor, P, K, N. \& Ho, G, W. Plasmonic photothermic directed broadband sunlight harnessing for seawater catalysis and desalination. Energy Environ. Sci. 9, 3151-3160 (2016).

[21] Zhou, L. et al. 3D self-assembly of aluminum nanoparticles for plasmon-enhanced solar desalination. Nat. Photonics 10, 393-398 (2016).

[22] Zhou, X. et al. Architecting highly hydratable polymer networks to tune the water state for solar water purification. Sci. Adv. 5, eaaw5484 (2019).

[23] Zhao, F. et al. Highly efficient solar vapour generation via hierarchically nanostructured gels. Nat. Nanotech. 13, 489-495 (2018).

[24] Chen, C. et al. Highly flexible and efficient solar steam generation device. Adv. Mater. 29, 1701756 (2017).

[25] Zhang, L. et al. Hydrophobic light to heat conversion membranes with self-healing ability for interfacial solar heating. Adv. Mater. 27, 4889-2894 (2015).

[26] Luo, J. et al. Aggregation-induced emission of 1-methyl-1,2,3,4,5-pentaphenylsilole. Chem. Commun. 18, 1740-1741 (2001).

[27] Mei, J. et al. Aggregation-Induced Emission: Together We Shine, United We Soar! Chem. Rev. 115, 11718-11940 (2015).

[28] Wang, D. et al. Boosting non-radiative decay to do useful work: development of a multi-modality theranostic system from AIEgen. Angew. Chem. Int. Ed. 11, 1510-1519 
(2018).

[29] Zhao, Z. et al. Highly efficient photothermal nanoagent achieved by harvesting energy via excited-state intramolecular motion within nanoparticles. Nat. Commun. 10, 768-779 (2019).

[30] Liu, S. et al. Molecular Motion in the Solid State. ACS Mater. Lett. 1, 425-431 (2019).

[31] Wang, K. et al. Aggregation induced emission fluorogens based nanotheranostics for targeted and imaging-guided chemo-photothermal combination therapy. Small 12, 6568-6575 (2016).

[32] Feng, G. \& Liu, B. Aggregation-induced emission (AIE) Dots: emerging theranostic nanolights. Acc. Chem. Res. 51, 1404-1414 (2018).

[33] Zhu, L. et al. Shape conformal and thermal insulative organic solar absorber sponge for photothermal water evaporation and thermoelectric power generation. Adv. Energy Mater. 9, 1900250 (2019).

[34] Ghasemi, H et al. Solar steam generation by heat localization. Nat. Commun. 5, 4449-4456 (2014).

[35] Liu, Y. et al. Bioinspired bifunctional membrane for efficient clean water generation. ACS Appl. Mater. Inter. 8, 772-779 (2016).

[36] Kuang, Y. et al. A high-performance self-regenerating solar evaporator for continuous water desalination. Adv. Mater. 31, 1900498 (2019).

[37] Zhu. M. et al. Plasmonic wood for high-efficiency solar steam generation. Adv. Energy Mater. 4, 1701028 (2018).

[38] Zhu, M. et al. Tree-inspired design for high-efficiency water extraction. Adv. Mater. 29, 1704107 (2017).

[39] $\mathrm{Xu}, \mathrm{N}$. et al. Mushrooms as efficient solar steam-generation devices. Adv. Mater. 29, 1606762 (2017).

[40] Guo, Y. et al. Synergistic energy nanoconfinement and water activation in hydrogels for efficient solar water desalination. ACS Nano 13, 7913-7919 (2019). 
[41] Zhou, X. et al. A hydrogel-based antifouling solar evaporator for highly efficient water desalination. Energy Environ. Sci. 11, 1985-1992 (2018).

[42] He, S. et al. Nature-inspired salt resistant bimodal porous solar evaporator for efficient and stable water desalination. Energy Environ. Sci. 12, 1558-1567 (2019).

[43] Xia, Y. et al. Spatially isolating salt crystallization from water evaporation for continuous solar steam generation and salt harvesting. Energy Environ. Sci. 12, 1840-1847 (2019).

[44] Jiang, P. et al. Poly(butylene terephthalate) fiber assembly with controllable pore size and gradient wettability: potential in simplifying cell culture procedure. ACS Macro lett. 7, 1192-1197 (2019)

[45] $\mathrm{Si}, \mathrm{Y}$. et al. Ultralight nanofiber-assemble cellular aerogels with superelasticity and multifunctionality. Nat. Commun. 5, 5802-5811 (2014).

[46] $\mathrm{Xu}, \mathrm{W}$. et al. Flexible and salt resistant Janus absorbers by electrospinning for stable and efficient solar desalination. Adv. Energy Mater. 8, 1702884 (2018).

[47] Yang, Y. et al. A general salt-resistant hydrophilic/hydrophobic nanoporous double layer design for efficient and stable solar water evaporation distillation. Mater. Horiz. 5, 1143-1150 (2018).

[48] Wang, X, Y. et al. Anti-biofouling double-layered unidirectional scaffold for long-term solar-driven water evaporation. J. Mater. Chem. A. 7, 16696-16703 (2019).

[49] Ren, H. et al. Hierarchical graphene foam for efficient omnidirectional solar-thermal energy conversion. Adv. Mater. 29, 1702590 (2017).

\section{Acknowledgements}

This work was supported by the National Natural Science Foundation of China (Grant No. 21801169), and Natural science foundation of Shenzhen University (Grant No. 2019004).

\section{Author contributions}

H. L., D. W., and B. Z. T. conceived and designed the experiments. H. W. and J. L. performed 
the synthesis AIE agent. H. L. conducted the fabrication, collected and analyzed the data. J. W assisted H. L. performed solar steam generation test. H. L., D. W., and B. Z. T co-wrote the paper. All the authors contributed to revise the manuscript.

\section{Additional information}

Supplementary Information accompanies this paper at https://

Competing interests: The authors declare no competing interests.

Reprints and permission information is available online at http://npg.nature.com/ reprintsandpermissions/

Publisher's note: Springer Nature remains neutral with regard to jurisdictional claims in published maps and institutional affiliations. 


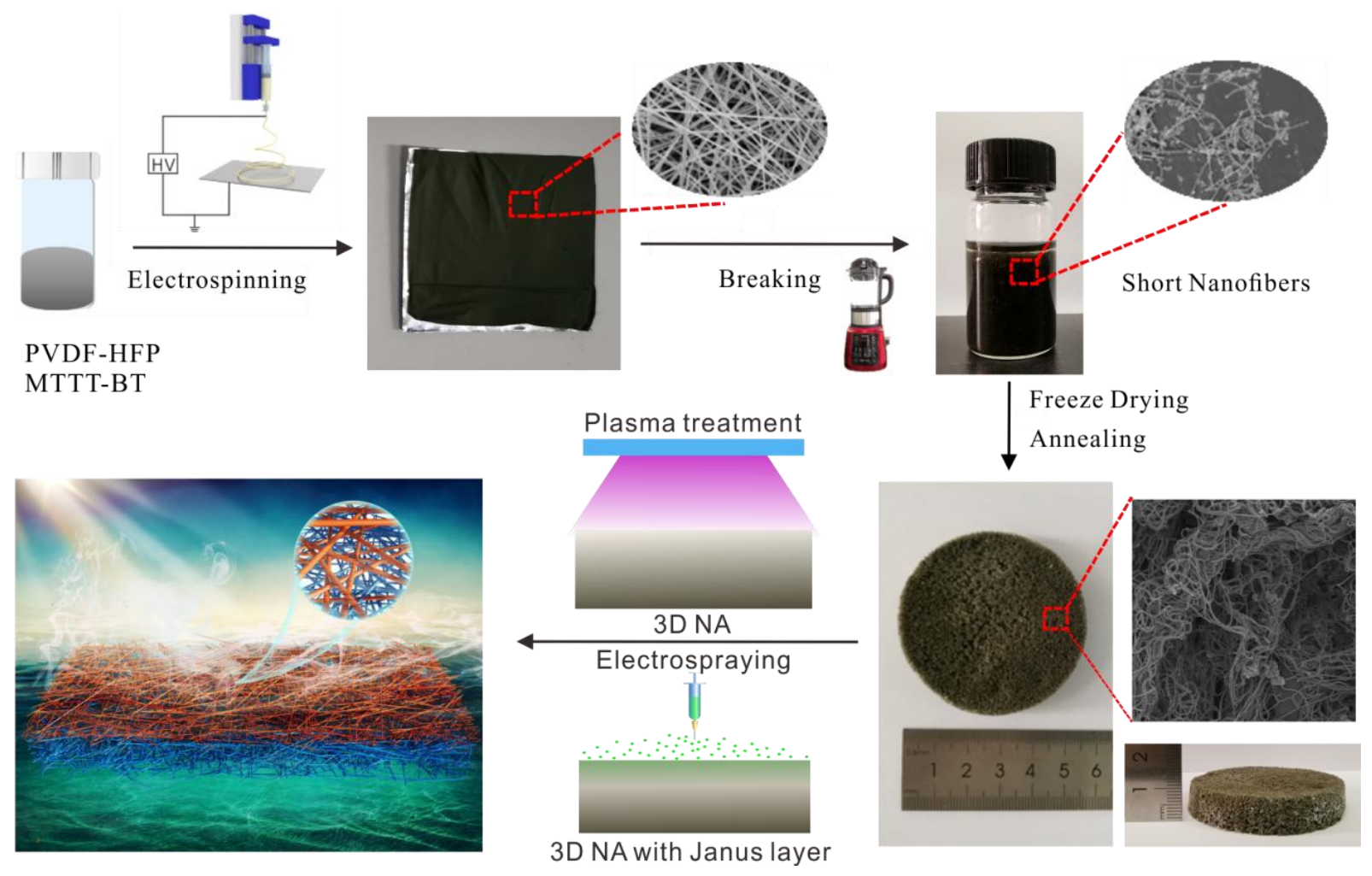

Fig. 1 Schematic of solar generation and salt harvesting. The photothermal agent was doped into PVDF-HFP solution and then electrospun into nanofibrous mat. The photothermal short nanofibers suspension made by high-speed blender and 3D nanofibrous aerogel (3D NA) were prepared by freeze drying the short nanofibers dispersions and thermal annealing treatment. The hydrophilic layers were achieved by $\mathrm{O}_{2}$ plasma active single layer and then electrosprayed by gelatin solution, the reverse layer was intrinsic hydrophobic due to the PVDF-HFP. 
A
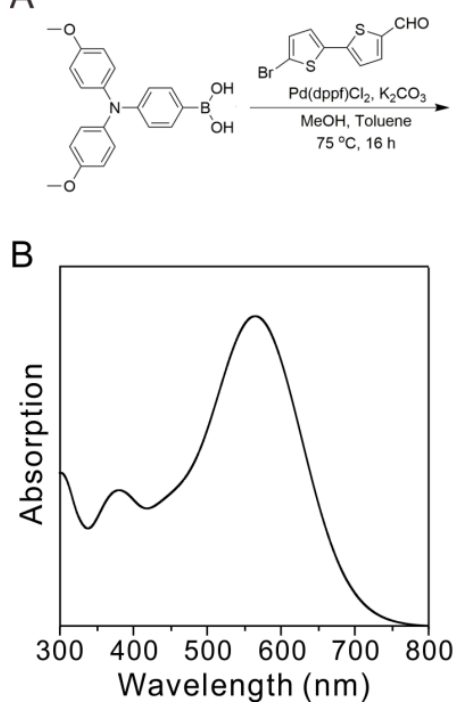

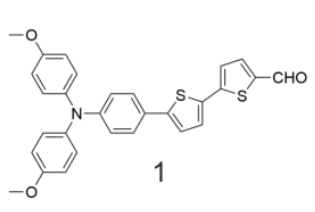

C

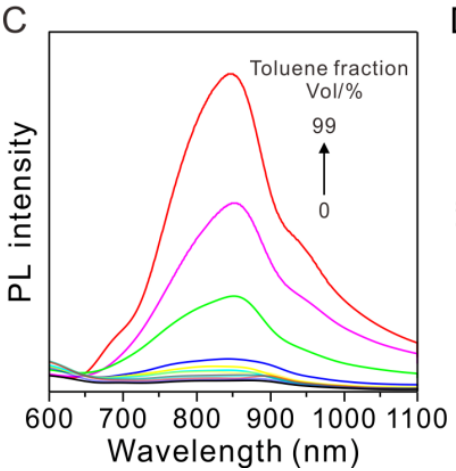

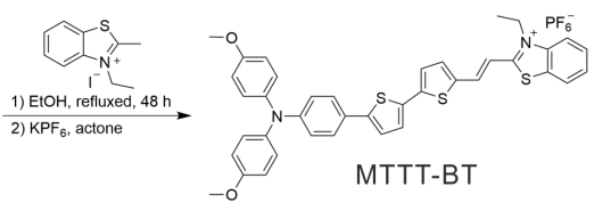

D

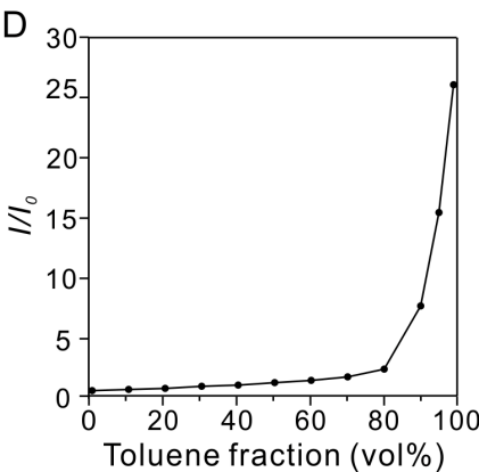

Fig. 2 (A) Synthetic route to MTTT-BT. (B) Absorption spectra of MTTT-BT in the DMSO solution. (C) PL spectra of MTTT-BT $\left(10 \times 10^{-6} \mathrm{M}\right)$ in DMSO/toluene mixture with different toluene fraction. (D) The plot of the relative emission intensity $\left(I / I_{0}\right)$ versus the composition of the solvent mixture. 

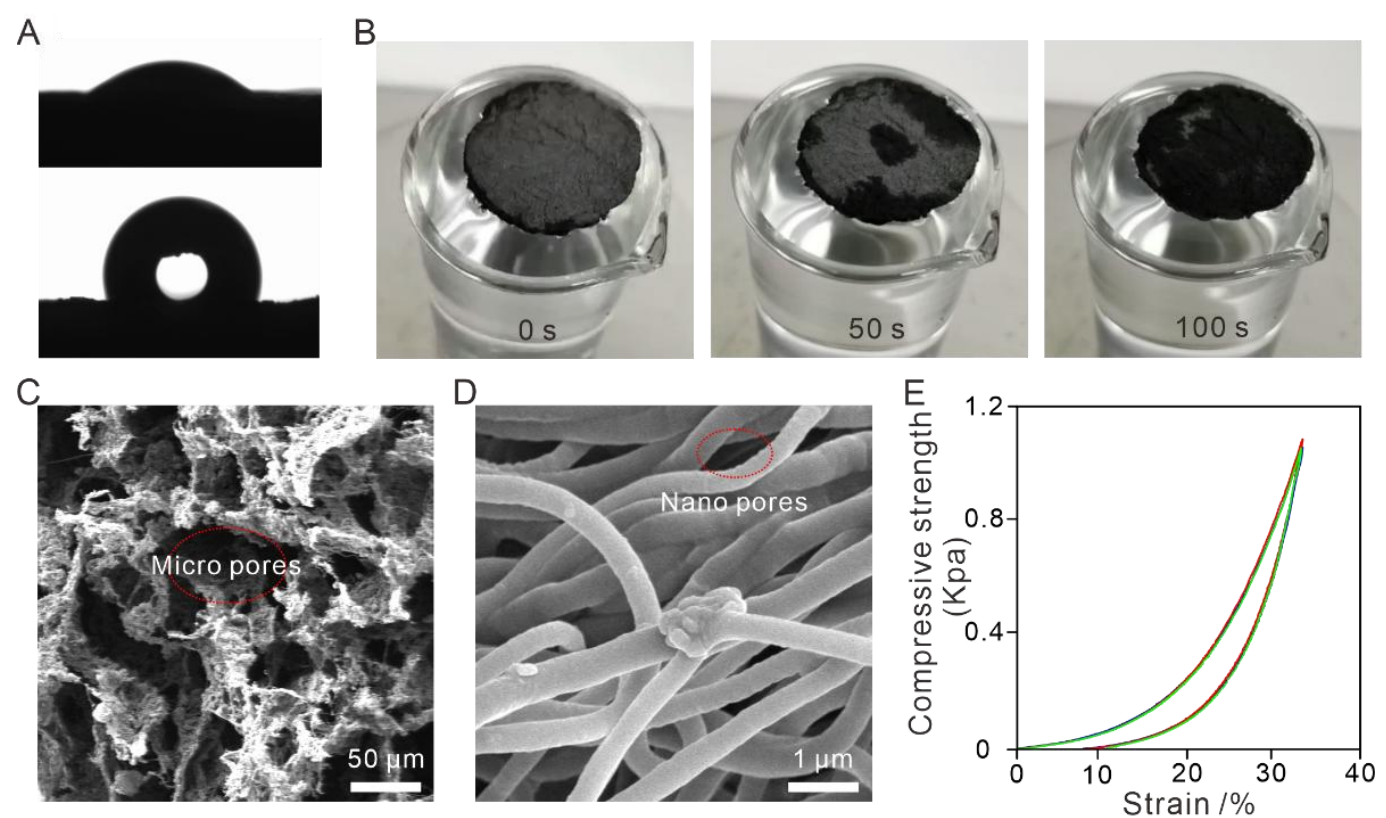

$\mathrm{F}$

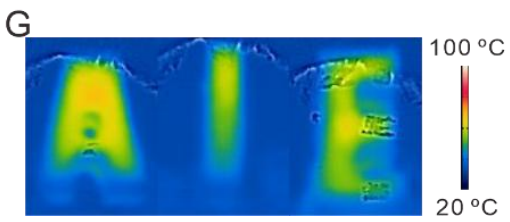

$\mathrm{H}$
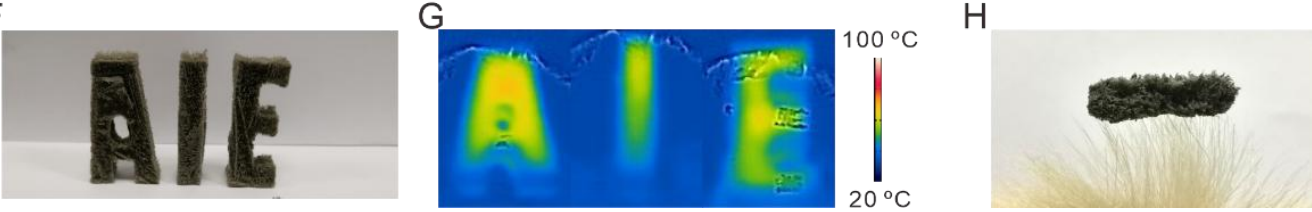

Fig. 3 (A) Water contact angle measurement capturing a water droplet on upper layer of 3D NA, and a water droplet standing on under layer of 3D NA, exhibited anisotropic wettability to water on its two sides. (B) Photo images of the 3D NA with Janus layers floating on the surface of water, the water was then pumped up and diffused to the entire surface of the 3D NA by hydrophilic interaction. (C, D) Microscopic architecture of 3D NA at various magnifications, showing the hierarchical porous structure. (E) Cyclic compressive results of the 3D NA. (F) An optical photograph of 3D NA with diverse shapes and (G) IR thermal images of 3D NA under irradiation of 1 sun. $(\mathrm{H}) \mathrm{A} 1 \mathrm{~cm}^{3} 3 \mathrm{D}$ NA $\left(p=10 \mathrm{mg} / \mathrm{cm}^{3}\right)$ stands on the rabbit feathers. 

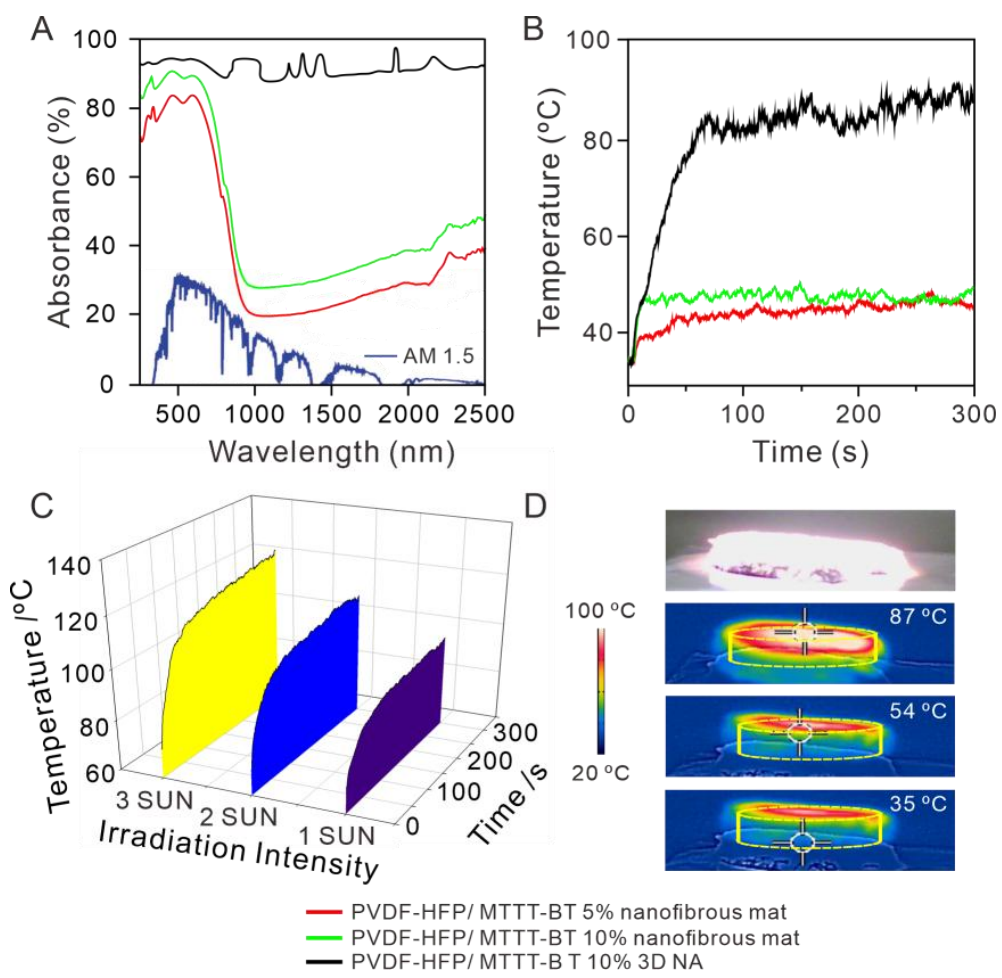

Fig. 4 (A) The absorption spectra of the 3D NA across 250-2500 nm, and solar spectral irradiance weighted by standard AM $1.5 \mathrm{G}$ solar spectrum. (B) The temperature changes of the 3D NA and the nanofibrous mat with different concentration of MTTT-BT as they were exposed from 1 sun $\left(1 \mathrm{KW} / \mathrm{m}^{2}\right)$ irradiation. (C) A comparison of the temperature rise recorded from the 3D NA upon the irradiation of the $1,2\left(2 \mathrm{KW} / \mathrm{m}^{2}\right)$ and $3 \mathrm{sun}\left(3 \mathrm{KW} / \mathrm{m}^{2}\right)$ for $5 \mathrm{~min}$. (D) IR thermal images showing the temperatures of the 3D NA (without any thermal insulation) at different position, the yellow dashed lines are the approximate edges of the 3D NA. 

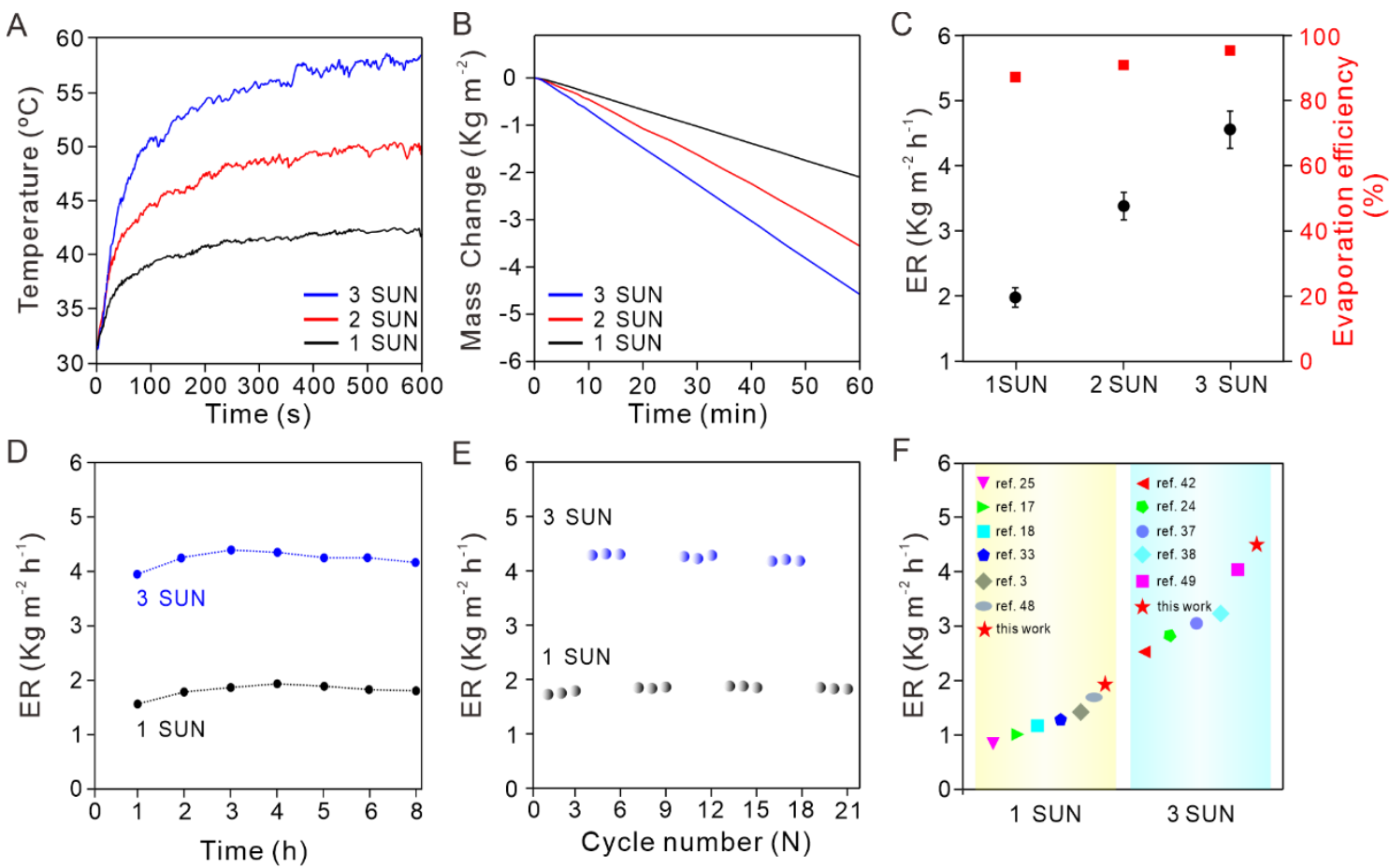

Fig. 5 (A) Plot showing the temperature of the surface, (B) the mass loss of water and (C) Evaporation rates (left-hand side axis) and corresponding energy conversion efficiencies (right-hand side axis) of 3D NA under different illumination intensity. (D) Evaporation rate of 3D NA over time. (E) The long-term stability of 3D NA, each cycle was sustained for $3 \mathrm{~h}$. (F) Comparison of 3D NA vapor generation performance and previous reports under 1 and 3 sun respectively. 

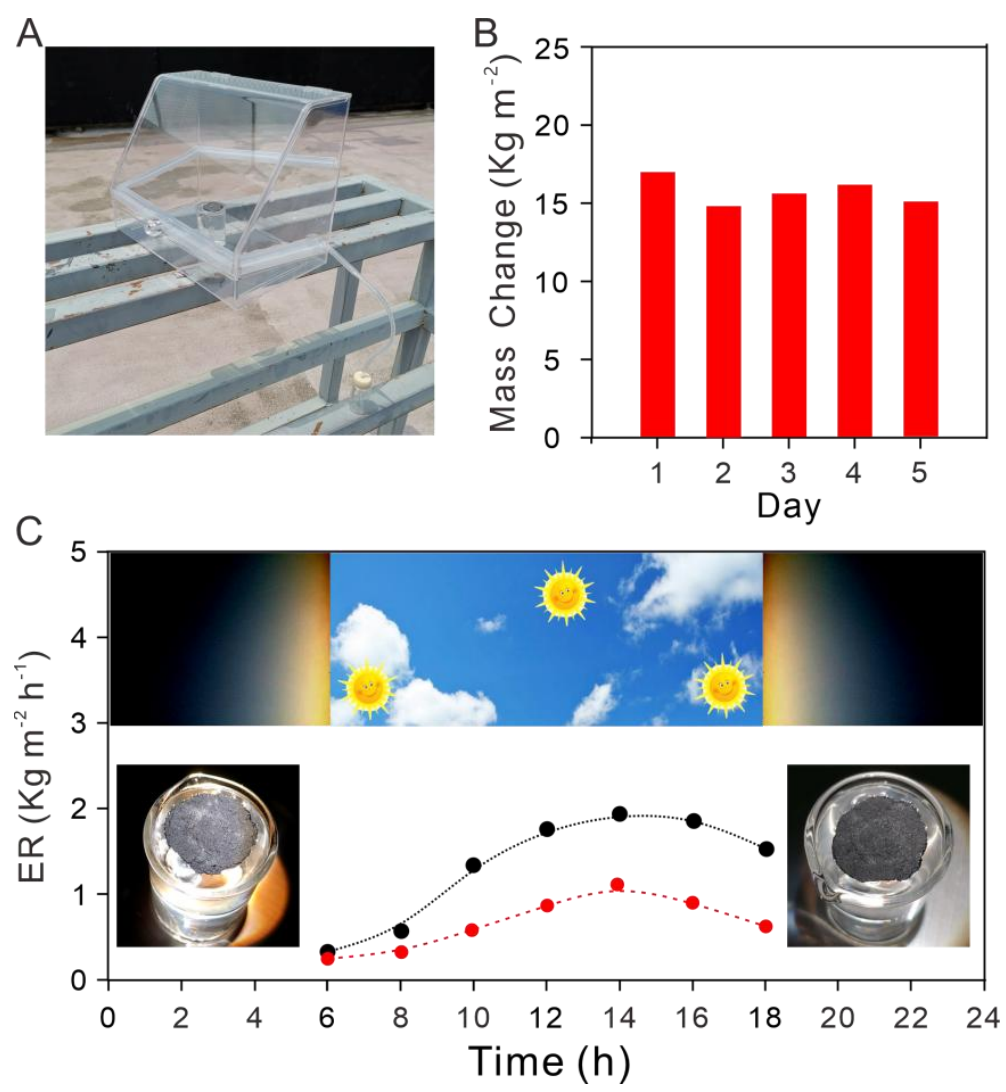

Fig. 6 (A) Photograph of hand-mand solar water evaporation device $(30 \times 15 \times 20 \mathrm{~cm})$, a 100 mL beaker contained $95 \mathrm{~mL}$ aqueous solution of $\mathrm{NaCl}$ with 3.5 wt.\% was placed in it. (B) The solar steam sustainability performance of an 3D NA under ambient environment (1 sun) for 5 days. (C) Evaporation rate of 3D NA (black spots) at 6:00 to 18:00 for over a day (red spots showed the evaporation rate of blank beaker without 3D NA). The inset showed photograph images of the 3D NA at begin and end under irradiation of sunlight for one day, no salt deposition was observed. 